\title{
OSMOTIC PUMP DRUG DELIVERY SYSTEM: A NOVAL APPROACH
}

\author{
Kashmir Singh*, Manpreet kaur Walia, Dr. Geeta agarwal, Dr. S. L. Harikumar \\ Rayat Bahra Institute Of Pharmacy VPO Sahauran Tehsil Kharar Distt. Mohali(Punjab) \\ *Corresponding Author's E mail: kashmirsingh940@gmail.com
}

\begin{abstract}
Conventional drug delivery systems have little control over their drug release and almost no control over the effective concentration at the target site. The major problem associated with conventional drug delivery system is unpredictable plasma concentrations. Controlled drug delivery systems offer spatial control over the drug release. Osmotic pumps are most promising systems for controlled drug delivery. These systems are used for both oral administration and implantation. The present review is concerned with the study of drug release systems which are tablets coated with walls of controlled porosity. . Osmotic pump uses the basic principle of osmosis for release of drug(s). Osmotic pumps consist of an inner core containing drug and osmogens, coated with a semi permeable membrane. As the core absorbs water, it expands in volume, which pushes the drug solution out through the delivery ports. Osmotic pumps release drug at a rate that is independent of the $\mathrm{pH}$ and hydrodynamics of the dissolution medium. Various patents available for osmotic drug delivery system like Rose-Nelson pump, Higuchileeper pump, higuchi-theeuwes pump and elementary osmotic pump. In this paper, various types of osmotic pump and the basic components of osmotic system tablets have been discussed briefly.
\end{abstract}

Keywords: Osmosis, component of osmotic system, Osmotic pump

\section{INTRODUCTION}

For many decades treatment of an acute disease or a chronic illness has been mostly accomplished by delivery of drugs to patients using various pharmaceutical dosage forms.Traditionally, the oral drug delivery has been popular as the most widely utilized route ofadministration among all the routes that have been explored for the systemic delivery of drugs. Conventional oral drug delivery systems are known to provide an immediate release of drug, in which one cannot control the release of the drug and cannot maintain effective concentration at the target site for longer time. The bioavailability of drug from these formulations may vary significantly, depending on factors such as physico-chemical properties of the drug, presence of excipient, various physiological factors such as the presence or absence of food, $\mathrm{pH}$ of the GI tract and GI motility. To overcome this limitation of oral route is replied by parenteral route. This route offers the advantage of reduced dose, targeting of site and avoiding GI stability, hepatic by-pass of drug molecule. In the recent years, pharmaceutical research has led to the development of several novel drug delivery systems. The role of drug development is to take a therapeutically effective molecule with sub-optimal physicochemical and/or physiological properties and develop an optimized product that will still be therapeutically effective but with additional benefits such $\mathrm{a}^{1}$.

Sustained and consistent blood levels within the therapeutic window

Enhanced bioavailability

Reduced interpatient variability

Customized delivery profiles

Decreased dosing frequency

Improved patient compliance Reduced side effects

C 2011, JDDT. All Rights Reserved
The drug release can be modulated by different ways but the most of novel drug delivery systems are prepared using matrix, reservoir or osmotic principle. In matrixsystems, the drug is embedded in a polymer matrix and the release takes place by partitioning of drug into the polymer matrix andthe surrounding medium. In contrast, reservoirsystems have a drug core surrounded by a rate controlling membrane. The osmotic systems utilize the principles of osmotic pressure for the delivery of drugs in both the routes oral as well as parenteral ${ }^{2}$

\section{ADVANTAGES}

Osmotic pump based drug delivery is formulated due to its many benefits over conventional dosage forms, some of which are as follows ${ }^{3-5}$ :

- The release rate of osmotic systems is highly predictable and can be programmed by modulating the release control parameters.

- The delivery rate of zero-order is achievable with osmotic systems.

- In the osmotic pump tablet frequency of dosing is reduced due to drug being released over a longer period of time unlike conventional tablets

- Extended release of a large amount of highly watersoluble drug by utilizing counter polymer in polyethylene oxides

- This is extremely valuable for patients with chronic illnesses which require the plasma concentrations of a drug to be within its therapeutic range to avoid breakthrough symptoms, for example, overnight management of pain in terminally ill patients

- The reduction or avoidance of side effects due to high plasma drug concentrations or 'dose dumping

ISSN: 2250-1177

CODEN (USA): JDDTAO 
- Better control of therapeutic drug concentration.

\section{DISADVANTAGES}

Development costs: Expensive specialized equipment and inert ingredients may be required for osmotic pump tablet formulations.

- Release rate: The drug release rate can be altered by food and gastric transit time; as a result differences may arise in the release rate between doses.

- Can not crush or chew products: Osmotic pump tablet should not be crushed or chewed as it can lead to loss of the 'slow release' characteristics as well as toxicity. ${ }^{6}$

\section{OSMOSIS}

Process of movement of the solvent from the lower concentration of solution to the higher concentration of the solution through the semipermiable membrane. Osmosis is the process that can control the drug delivery system. Osmotic pressure created due to imbibitions of fluid from external environment into the dosage form regulates the delivery of drug from osmotic device. Rate of drug delivery from osmotic pump is directly proportional to the osmotic pressure developed due to imbibitions of fluids by osmogen. Osmotic pressure is a colligative property of a solution in which the magnitude of osmotic pressure of the solution is independent on the number of discrete entities of solute present in the solution. Hence the release rate of drugs from osmotic dispensing devices is dependent on the solubility and molecular weight and activity coefficient of the solute (osmogent). ${ }^{7}$

\section{Principal f Osmosis}

The first report of an osmotic effect dates to Abbenollet (1748). But Pfeffer obtained the first quantitative measurement in 1877. In Pfeffer experiment a membrane permeable to water but impermeable to sugar is used to separate a sugar solution from pure water. A flow of water then takes place into the sugar solution that cannot be halted until a pressure $\pi$ is applied to the sugar solution. Pfeffer showed that this pressure, the osmotic pressure $\pi$ of the sugar solution is directly proportional to the solution concentration and the absolute temperature. Within few years, Vant Hoff had shown the analogy between these results and ideal gas laws by the expression

$\pi=\Phi$ c r t

Where $\Phi$ is the osmotic coefficient of the solution, $\mathrm{c}$ is the molar concentration of sugar in the solution, $r$ is the gas constant, $\mathrm{t}$ is the absolute temperature.

Osmotic pressure for concentrated solution of soluble solutes commonly used in controlled release formulation are extremely high ranging from 30 atm for sodium phosphate up to $500 \mathrm{~atm}$ for a lactose-fructose mixture, as their osmotic pressure can produce high water flow across semi permeable membrane. The osmotic water flow through a membrane is given by the equation

$\mathrm{Dv} / \mathrm{dt}=\mathrm{A} \mathrm{Q} \Delta \pi / \mathrm{L}$

Where dvldt is water flow across the membrane of area A, thickness L, and the permeability
$\mathrm{Q}$ in $\mathrm{cm} 2, \Delta \pi$ is the osmotic pressure difference between the two solutions on either side of the membrane.

This equation is strictly for completely perm selective membrane that is membrane permeable to water but completely impermeable to osmotic agent. ${ }^{8}$

\section{Basic Component of Osmotic System}

- Drug

- Osmotic agent

- Semipemiable membrane

- Wicking agent

- Pore forming agent

- Coating agent

\section{Drug}

All drugs are not suitable for osmotic system as prolong action medication .Drugs those which has biological half-life more than $12 \mathrm{hr}$ e.g.: Diazepam and drug which have very short half life i.e. less than $1 \mathrm{hr}$ e.g. Penicillin $G$, furosemide are not suitable candidate for osmotic controlled release. Drug which have biological half-life in between $1-6 \mathrm{hrs}$ and which is used for prolonged cure of diseases are ideal applicant for osmotic systems. ${ }^{9}$

Drug having following characteristics are suitable for formulation

1. It should have short half-life

2. Prolonged release of drug should be desired.

3. It should be potent in nature.

4. Solubility of drug should not be very high or very low. 10

\section{Osmotic agent}

These are also known as osmogens or osmogents and are used to create osmotic pressure inside the system. When the solubility of drug is low then the drug will show zero order release but at a slow rate. To enhance the release rate osmotic agent is added in the formulation. Osmotic agent creates a very high osmotic pressure gradient inside the system and increases release rate of drug. ${ }^{11}$

\section{Some of the commercially used osmotic agents}

Sodium chloride, Fructose, sucrose, Potassium chloride, Xylitol, Sorbitol, citric acid, Dextrose, Manitole and Lactose.

\section{Some Mixture Used As a Osmotic Agent}

- Dextrose +Fructose

- $\quad$ Lactose +Fructose

- Sucrose+ Fructose

- Lactose +Dextrose

- Mannitol +Fructose

- Mannitol +Dextrose

- Dextrose + Sucrose

- Mannitol +Sucrose $\mathrm{S}^{12}$ 


\section{Semi permeable Membrane}

Since the membrane in osmotic systems is semi permeable in nature, any polymer that is permeable to water but impermeable to solute can be selected. ${ }^{13}$ Cellulose acetate is a commonly employed semi permeable polymer for the preparation of osmotic pumps. It is available in different acetyl content grades. Particularly, acetyl content of $32 \%$ and $38 \%$ are widely used. Acetyl content is described by the degree of substitution (DS), i.e. the average number of hydroxyl groups on the anhydroglucose unit of the polymer replaced by substituting group. Some of the polymers that can be used for above purpose include cellulose esters such as cellulose acetate, cellulose diacetate, cellulose triacetate, cellulose propionate, cellulose acetate butyrate, and cellulose ethers like ethyl cellulose. ${ }^{14}$ The Semi Permeable Membrane must meet some performance criteria;

- $\quad$ The material must possess sufficient wet strength (105 ) and wet modulus so as to retain its dimensional integrity during the operational lifetime of the device.

- The membrane exhibit sufficient water permeability so as to retain water flux rate in the desired range. The water vapor transmission rates can be used to estimate water flux rates.

- The reflection coefficient and leakiness of the osmotic agent should approach the limiting value of unity. Unfortunately, polymer membranes that are more permeable to water are also, in general more permeable to the osmotic agent.

- The membrane should also be biocompatible. ${ }^{15}$

\section{Wicking agent}

The wicking agents are those agents which help to increase the contact surface area of the drug with the incoming aqueous fluid. The use of the wicking agent help to enhance the rate of drug released from the orifice of the drug. The examples are colloidal silicon dioxide, PVP \& Sodium laryl sulphate. ${ }^{16}$

\section{Pore Forming Agents}

The pore-forming agents cause the formation of micro porous membrane. The micro porous wall may be formed in situ by a pore-former by its leaching during the operation of the system. The pore-formers can be inorganic or organic and solid or liquid in nature. For example, alkaline metal salts such as sodium chloride, sodium bromide, potassium chloride, potassium sulphate, potassium phosphate etc., alkaline earth metals such as calcium chloride and calcium nitrate, carbohydrates such as sucrose, glucose, fructose, mannose, lactose, sorbitol, and mannitol and, diols and polyols such as poly hydric alcohols, polyethylene glycols and polyvinyl pyrrolidone can be used as pore forming agents. ${ }^{17}$

\section{Coating solvents}

The primary function of solvent system is to dissolved or dispersed the polymer and other additive and convey them to substrate surface. solvent used to prepare polymeric solution include inert inorganic and organic solvents that do not adversely harm the core, wall and other material the various types of solvents and their combinations are as follows: Methylene chloride, methanol, isopropyl alcohol, dichloromethane, ethyl acetate, acetone, carbon tetrachloride, cyclohexane, butyl alcohol, water etc and the mixture of solvents such as acetone-methanol(80:20), methylene chloride- methanol (79:21), acetone-ethanol(80:20), methylene chloridemethanol-water $(75: 22: 3)^{18}$

\section{Mechanism of drug release}

Tablet has rigid water permeable jacket with one or more laser dried small holes. As the tablet passes through the body the osmotic pressure of the tablet pushes the active drug through the opening in the tablet. The basic equation which applies to osmotic systems is

$$
\begin{gathered}
d M / d t=d V / d t . c \ldots \ldots . . .(a) \text { Where }, \\
d M / d t=\text { mass release } \\
d V / d t=\text { volumetric pumping rate } \\
c=\text { concentration of drug But, } \\
d V / d t=(A / h) L p \\
(\sigma \Delta \Pi-\Delta p) \text { Where, }
\end{gathered}
$$

$\mathrm{A}=$ membrane area, $\mathrm{h}=$ thickness of membrane , $\mathrm{Lp}=$ mechanical permeability,$\sigma=$ reflection coefficient ,$\Delta \Pi=$ osmotic pressure difference,$\Delta \mathrm{p}=$ hydrostatic pressure difference

As the size of orifice delivery increases.

$\Delta \mathrm{p}$ decrease, so $\Delta \Pi>\Delta \mathrm{p}$ and equation becomes $\mathrm{dV} / \mathrm{dt}$ $=\mathrm{A} / \mathrm{h} \operatorname{Lp}(\sigma \Delta \Pi)$ When the osmotic pressure of the formulation is large compared to the osmotic pressure of the environment, $\mathrm{p}$ can be substituted for $\mathrm{Dp}$.

$$
\begin{gathered}
d V / d t=A / h L p \\
\sigma \Pi=A / h k \Pi
\end{gathered}
$$

$(\mathrm{k}=\mathrm{Lp} \sigma=$ membrane permeability), Now, equation (a) can be given as

$\mathrm{dM} / \mathrm{dt}=(\mathrm{A} / \mathrm{h}) \mathrm{k} \Pi \mathrm{c}=(\mathrm{A} / \mathrm{h}) \mathrm{k} \Pi \mathrm{S}(\mathrm{S}=$ solubility of drug, c taken as $\mathrm{S})$

\section{Osmotic pump system :}

Table 1: Classification of Osmotic Pump Drug Delivery System ${ }^{19}$

\begin{tabular}{|l|l|l|}
\hline Implantable & Oral osmotic Pump & Specific types \\
\hline The Rose and Nelson Pump & Single chamber osmotic pump: & Controlled porosity osmotic pump, \\
\hline Higuchi Leeper Pump & Multi chamber osmotic pump: & Osmotic bursting osmotic pump, \\
\hline Higuchi Theuwes pump & $\begin{array}{l}\text { Push pull osmotic pump, } \\
\text { Osmotic pump with non-expanding } \\
\text { second chamber }\end{array}$ & Liquid OROS, \\
\hline
\end{tabular}




\begin{tabular}{|l|l|l|}
\hline Implantable Mini osmotic pump & & Delayed Delivery osmotic system \\
\hline & & OROS-CT (colon targeting), \\
\hline & & sandwiched oral therapeutic system, \\
\hline & & Osmotic pump for insoluble drugs, \\
\hline & & Monolithic osmotic system and OSMAT \\
\hline
\end{tabular}

\section{Implantable Pump}

\section{The Rose and Nelson Pump}

In, 1955, two Australian physiologists reported the first osmotic pump. They were interested in delivery of drug to the gut of sheep and cattle. The pump consisted of three chambers a drug chamber with an orifice, a salt chamber with elastic diaphragm containing excess solid salt, and a water chamber. A semipermiable membrane separates the drug and water chamber. The difference in osmotic pressure across the membrane moves water from the water chamber in to the salt chamber. The volume of chamber increases because of this water flow, which distends the latex diaphragm separating the salt and drug chambers, thereby pumping drug out of the device $\mathrm{e}^{20}$

\section{Higuchi Leeper Pump}

Higuchi Leeper pump is widely swallowed or implanted in the body of animal for delivery of antibiotic or growth hormones. Higuchi Leeper pump consist of rigid housing and semi permeable membrane. A layer of low melting waxy solid, such as microcrystalline paraffin wax is used in place of elastic diaphragm to separate the drug and osmotic chamber. Recent modification in HiguchiLeeper pump accommodated pulsatile drug delivery. The pulsatile release was achieved by the production of a critical pressure at which the delivery orifice opens and releases the drug. ${ }^{21}$ Pulsatile delivery could be achieved by using Higuchi Leeper pump; such modifications are described and illustrated in Figure. The Pulsatile release of drug is achieved by drilling the orifice in elastic material that stretches under the osmotic pressure. Pulse release of drug is obtained after attaining a certain critical pressure, which causes the orifice to open. The pressure then reduces to cause orifice closing and the cycle repeats to provide drug delivery in a pulsatile fashion. The orifice should be small enough to be substantially closed when the threshold level of osmotic pressure is not present ${ }^{22}$

\section{Higuchi-Theeuwes pump}

In the early 1970s, Higuchi and Theeuwes6 developed another, even simpler variant of the Rose-Nelson pump. As with the Higuchi- Leeper pump, water to activate the osmotic action of the pump is obtained from the surrounding environment. In the Higuchi-Theeuwes device, however, the rigid housing is dispensed with and the membrane acts as the outer casing of the pump. This membrane is quite sturdy and is strong enough to withstand the pumping pressure developed inside the device. The device is loaded with the desired drug prior to use. When the device is placed in an aqueous environment, release of the drug follows a time course set by the salt used in the salt chamber and the permeability of the outer membrane casing. Most of the Higuchi-Theeuwes pumps use a dispersion of solid salt in a suitable carrier for the salt chamber of the device ${ }^{23}$

\section{Implantable Mini osmotic pump}

Implantable Mini osmotic pump shown in figure 3 it is composed of three concentric layers-the drug reservoir, the osmotic sleeves and the rate controlling semi permeable membrane. The additional component called flow moderator is inserted into the body of the osmotic. The inner most compartment of drug reservoir which is surrounded by an osmotic sleeve, a cylinder containing high concentration of osmotic agent. The osmotic sleeve is covered by a semi permeable membrane when the system is placed in aqueous environment water enters the sleeve through semi permeable membrane, compresses the flexible drug reservoir and displaces the drug solution through the flow moderator. These pumps are available with variety of delivery rates between 0.25 to $10 \mathrm{ml}$ per hour and delivery duration between one day and four weeks $^{24}$

\section{Single chamber osmotic pump:-}

\section{Elementary osmotic pump :-}

Elementary osmotic pump was invented by Theeuwes in 1974 and it essentially contains an active agent having a suitable osmotic pressure, it is fabricated as a tablet coated with semi permeable membrane, usually cellulose acetate. ${ }^{25}$ A small orifice is drilled through the membrane coating. (When this coated tablet is exposed to an aqueous environment, the osmotic pressure of the soluble drug inside the tablet draws water through the semipermeable coating and a saturated aqueous solution of drug is formed inside the device. The membrane is non-extensible and the increase in volume due to inhibition of water raises the hydrostatic pressure inside the tablet, eventually leading to flow of saturated solution of active agent out of the device through a small orifice.

The pump initially releases the drug at a rate given by equation;

$$
d M t / d t=(d V / d t) . C s \quad \text { Where, }
$$

$\mathrm{dV} / \mathrm{dt}$ depicts the water flow into the tablet

Cs is the solubility of the agent inside the tablet. 


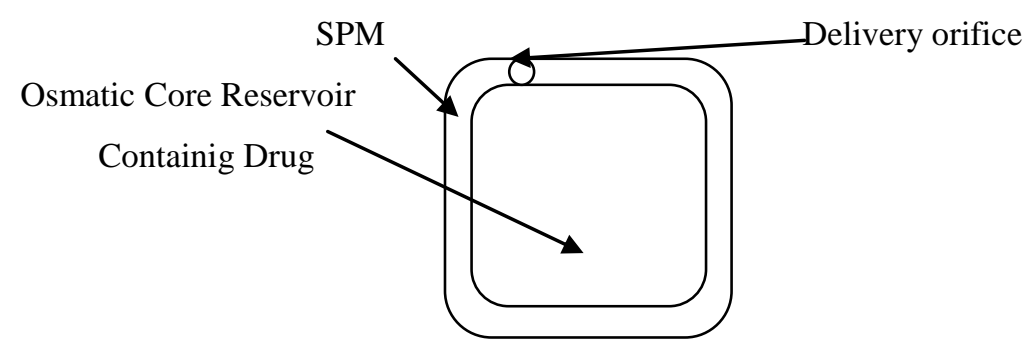

Fig 1: Elementary osmotic pump

\section{Multi chamber osmotic pump}

\section{A. Push pull osmotic pump}

Push pull osmotic pump is a modified EOP. Through, which it is possible to deliver both poorly water-soluble and highly water soluble drugs at a constant rate. This system resembles a standard bilayer coated tablet. One layer (depict as the upper layer) contains drug in a formulation of polymeric, osmotic agent and other tablet excipients. This polymeric osmotic agent has the ability to form a suspension of drug in situ. When this tablet later imbibes water, the other layer contains osmotic and colouring agents, polymer and tablet excipients. These layers are formed and bonded together by tablet compression to form a single bilayer core. The tablet core is then coated with semi permeable membrane. After the coating has been applied, a small hole is drilled through the membrane by a laser or mechanical drill on the drug layer side of the tablet. When the system is placed in aqueous environment water is attracted into the tablet by an osmotic agent in both the layers. The osmotic attraction in the drug layer pulls water into the compartment to form in situ a suspension of drug. The osmotic agent in the nondrug layer simultaneously attract water into that compartment, causing it to expand volumetrically and the expansion of non drug layer pushes the drug suspension out of the delivery orifice. ${ }^{26}$

\section{B. Osmotic Pump with Non Expanding Second Chamber}

\section{Specific types}

The second category of multi-chamber devices comprises system containing a non-expanding second chamber. This group can be divided into two sub groups, depending on the function of second chamber. In one category of these devices, the second chamber is used to dilute the drug solution leaving the devices. This is useful because in some cases if the drug leaves the oral osmotic devices a saturated solution, irritation of GI tract is a risk. Example: The problem that leads to withdrawal of osmosin, the device consists of a normal drug containing porous tablet from which drug is released as a saturated solution. However before the drug can escape from the device it must pass through a second chamber. Water is also drawn osmotically into this chamber either because of osmotic pressure of drug solution or because the second chamber contain, water soluble diluents such as $\mathrm{NaCl}$. This type of devices consist of two rigid chamber, the first chamber contains a biologically inert osmotic agent, such as sugar or a simple salt like sodium chloride, the second chamber contains the drug. In use water is drawn into both the chamber through the surrounding semi permeable membrane. The solution of osmotic agent formed in the first chamber then passes through the connecting hole to the drug chamber where it mixes with the drug solution before exiting through the micro porous membrane that form a part of wall surrounding the chamber. The device could be used to deliver relatively insoluble drugs $^{27}$

Table 2: Specific Types Osmotic Pump

\begin{tabular}{|l|l|c|}
\hline \multicolumn{1}{|c|}{ TYPE } & \multicolumn{1}{|c|}{ DESCRIPTION } \\
\hline $\begin{array}{l}\text { Controlled } \\
\text { porosity } \\
\text { osmotic pump }\end{array}$ & $\begin{array}{l}\text { The pump can be made with single or multicompartment } \\
\text { dosage form, in either form, the delivery system comprises a } \\
\text { core with the drug surrounded by a semipermeable membrane } \\
\text { which has an asymmetric structure. When exposed to water, } \\
\text { low levels of water-soluble additive are leached from } \\
\text { polymer materials that were permeable to water yet remained } \\
\text { insoluble. Then resulting sponge like structure formed the } \\
\text { controlled porosity walls of interest and was substantially } \\
\text { permeable to both water and dissolved drug agents. }\end{array}$ & $\begin{array}{l}\text { Semipermiable Membrane With Water } \\
\text { Soluble Additives }\end{array}$ \\
\hline $\begin{array}{l}\text { Osmotic } \\
\text { bursting } \\
\text { osmotic pump }\end{array}$ & $\begin{array}{l}\text { In this system delivery orifice is absent and size may be } \\
\text { smaller. When it is placed in an aqueous environment, water } \\
\text { is imbibed and hydraulic pressure is built up inside until the } \\
\text { wall rupture and the content are released to the environment }\end{array}$ & $\begin{array}{l}\text { SEMIPERALABLE MEMBRANE } \\
\text { Figure 2: controlled porosity pump }\end{array}$ \\
\hline
\end{tabular}




\begin{tabular}{|l|l|l|}
\hline Liquid OROS & $\begin{array}{l}\text { Liquid OROS are designed to deliver drugs as liquid } \\
\text { formulations and combine the benefits of extended release } \\
\text { with high bioavailability. They are of three types: a) L OROS } \\
\text { hard cap b) L OROS soft cap c) delayed liquid bolus delivery } \\
\text { system. 28 }\end{array}$ & $\begin{array}{l}\text { It is composed of polymeric push layer sandwiched between } \\
\text { two drug layers with two delivery orifices. }\end{array}$ \\
\hline $\begin{array}{l}\text { Sandwiched } \\
\text { oral therapeutic } \\
\text { system }\end{array}$ & $\begin{array}{l}\text { The device concerns an osmotic agent for dispensing } \\
\text { beneficial active agent that has poor solubility in water. The } \\
\text { for insoluble of the system comprises a beneficial amount of a } \\
\text { drugs pubstantially water- insoluble active agent, which is lipid } \\
\text { soluble or lipid- wettable; a sufficient amount of water } \\
\text { insoluble lipid carrier, which is liquid at the temperature of } \\
\text { use to dissolve or suspend the drug and agent to ensure the } \\
\text { release of the lipid carrier of the drug from the pump. 30 }\end{array}$ & \\
\hline
\end{tabular}

\section{EVALUATION PARAMETER OF OSMOTIC DRUG DELIVERY FORMULATION:-}

Characterization of dosage form

Effect of osmotic agents

Swelling properties

Membrane stability and thickness

Orifice diameter and drug release

In-vitro drug release study. ${ }^{31}$
The in vitro release of drugs from oral osmotic systems has been evaluated by the conventional USP paddle and basket type apparatus.

The dissolution medium is generally distilled water as well as simulated gastric fluid (for first 2-4 h) and intestinal fluids (for subsequent hours) have been used.

The standard specifications, which are followed for the oral controlled drug delivery systems are equivalently applicable for oral osmotic pumps.

In vivo evaluation of oral osmotic systems has been carried out mostly in dogs. Monkeys can also be used but in most of the studies the dogs are preferred. ${ }^{32}$

\section{MARKET PRODUCTS:-}

Table 3: Products Incorporating ALZA's OROS® Technology

\begin{tabular}{|l|l|l|}
\hline BRAND NAME & \multicolumn{1}{|c|}{ SALT } & \multicolumn{1}{c|}{ USED } \\
\hline Alpress ${ }^{\mathrm{TM}} \mathrm{LP}$ & prazosin & For the treatment of hypertension. \\
\hline Cardura $^{\circledR} \mathrm{XL}$ & doxazosin mesylate & for the treatment of hypertension \\
\hline Concerta & methylphenidate $\mathrm{HCl}$ & Attention Deficit Hyperactivity Disorder \\
\hline Covera-HS & verapamil & Management of hypertension and angina pectoris. \\
\hline Ditropan XL & oxybutynin chloride & $\begin{array}{l}\text { Overactive bladder. Symptoms of urge urinary incontinence, urgency and } \\
\text { frequency. }\end{array}$ \\
\hline DynaCirc $\mathrm{CR}^{\circledR}$ & isradipine & for the treatment of hypertension \\
\hline Efidac 24 & chlorpheniramine & Allergy symptoms and nasal congestion. \\
\hline Glucotrol XL ${ }^{\circledR}$ & glipizide & $\begin{array}{l}\text { for the control of hyperglycemia in patients with non-insulin-dependent } \\
\text { diabetes }\end{array}$ \\
\hline Sudafed ${ }^{\circledR} 24$ Hour & pseudoephedrine & nasal decongestant \\
\hline Procardia XL ${ }^{\circledR}$ & nifedipine & For the treatment of angina and hypertension. \\
\hline Volmax & albuterol & bronchospasm in patients with reversible obstructive airway disease \\
\hline
\end{tabular}

\section{CONCLUSION}

Osmotic pumps are the most reliable controlled drug delivery system. It uses osmotic pressure for controlled delivery of active agent. It allows targeted delivery of agents to virtually any tissue. It ensures around the clock exposure to test agent at predictable levels. Osmotic pumps have excellence control on the drug delivery so these are mostly used now a days. 


\section{REFERENCES}

1. Prescott L.F, The need for improved drug delivery in clinical practice, In: Novel Drug Delivery and Its Therapeutic application, John Wiley and Sons, West Susset, U.K. 1989: 1-11.

2. Bhatt PP. Osmotic drug delivery systems for poorly water soluble drugs, Pharmaventures Ltd., Oxford.

3. Kojima H, Yoshihara K, Sawada T, Kondo H and Sako K. 2008.

4. PEO)/polyethylene glycol (PEG) matrix tablets. Eur. J. Pharm. Biopharm, 70, 556-562.

5. Aulton's Pharmaceutics; The Design and Manufacture of Medicines. 3rd ed. Philadelphia, USA: Churchill Livingstone Elsevier. pp: 99-102.)

6. DiMatteo MR and DiNicola DD. 1982. Achieving patient compliance: the psychology of the medical practioners role. New York: Pergamon press.

7. Martin A. Phyical Pharmacy, 4th Edition, Lippincott Williams and Wilkins 1994; 116-117.

8. Santus G, Baker RW. J Control Release 1995; 35: 2. [ Pfefer WEP. Osmotishe Umtersuchen, Leipzig, 1877.

9.Gadwal P, Rudrawal P. International Journal of Pharmacy \& Life Sciences 2010; 1(6): 302-312.

10. Sharma S, Singh SP, Bhardwaj S, Gaurave K, Gupta GD. Latest Reviews [Internet]. 2008;6

11.16Rastogi SK, Vaya N, Mishra B. East Pharm 1995; 38: 79-82

12.Santus G., Baker RW. Controlled Release 1995; 35: 1-2 Tanmoy Ghosh, Amitava Ghosh. Journal of Applied Pharmaceutical Science 2011; 1 (2): 38-49

13. B Lindstedt, G Ragnarsson, J Hjartstam. Int J Pharm 1989; (56): 261-268.

14.Seminoff, GM Zentner. Cellulosic coating, US patent 5, $1992,126,146$

15. Tanmoy Ghosh, Amitava Ghosh. Journal of Applied Pharmaceutical Science 2011; 1 (2): 38-49

16.Tanmoy Ghosh, Amitava Ghosh. Journal of Applied Pharmaceutical Science 2011; 1 (2): 38-49
17.GM Zentner, GS Rork, KJ Himmelstein. US patent 4, $1990,968,507$

18. Rajagopal K, Nallaperumal N, Venkatesan S. Lipids in Health and Disease 2011; 10: 51

19.Stuti G, Ravindra PS, Rohitashva S. International Journal of Comprehensive Pharmacy 2011; 6: 1 .

20.NK Jain. Advances in controlled and novel drug delivery, CBS Publisher \& distributer, first adition. Page No. 20

21.Gadwal P, Rudrawal P. International Journal of Pharmacy \& Life Sciences 2010; 1(6): 302-312.

22.T Higuchi, HM Leeper. US Patent No. 399.

23.Higuchi, et al. US Patent 3995631 (1976)

24.Gadwal P, Rudrawal P. International Journal of Pharmacy \& Life Sciences 2010; 1(6): 302-312.

25. Ouyang D, Nie S, Li W, Guo H, Liu H, Pan W. J Pharm Pharmacol 2005; (57): 817-820.

26.Parma NS, Vyas SK, NK Jain. In: Advanced in controlled and novel drug delivery. CBS publisher, 28-29.

27.Srenivasa B, Kumar NR, Murthy KVR. Eastern Pharmacist $2001 ; 22$.

28.Javad Shokri, Parinaz Ahmadi, Parisa Rashidi, Mahbobeh Shahsavari, Ali Rajabi-Siahboomi, Ali Nokhodchi. European Journal of Pharmaceutics and Biopharmaceutics 2008; 68: 289297.

29. Liang C Dong, Patrick Wong, Jason Wan, Keru Shafi. Drug Delivery Technology 2002; 2 (1)

30.Longxiao Liu, Jeong $\mathrm{Ku}$, Gilson Khang, Bong Lee, John M Rhee, Hai Bang Lee. Journal of Controlled Release 2000; 68: 145-156.

31. Gaylen ZM, Gerald SR, Kenneth JH. J Control Release 1985; 1 : 269-282

32.RS Thakor, FD Majmudar, JK Patel and GC Rajaput. Journal of Pharmacy Research 2010; (34): 771-775 